\title{
Preference evaluation techniques of preference queries in database
}

\begin{abstract}
Preference queries are considered as a major necessity tool in today's database management system (DBMS). Adopting preference queries in the database application systems enable users to determine more than one objective in the submitted query which result into more accurate results compared to the traditional queries. Preference queries prefer one data item (tuple) $\mathrm{p}$ over the other data item (tuple) $\mathrm{q}$ if and only if $\mathrm{p}$ is better than $\mathrm{q}$ in all dimensions (attributes) and not worse than $\mathrm{q}$ in at least one dimension (attribute). Several preference evaluation techniques for preference queries have been proposed which aimed at finding the "best" results that meet the user preferences. These include but not limited to top-k, skyline, ranked skylines, $\mathrm{k}$-representative dominance, $\mathrm{k}$-dominance,top- $\mathrm{k}$ dominating, and $\mathrm{k}$ frequency. This paper attempts to survey and analyze the following preference evaluation techniques of query processing in database systems: top-k, skyline, top-k dominating, $\mathrm{k}-$ dominance, and k-frequency by highlighting the strengths and the weaknesses of each technique.
\end{abstract}

Keyword: Skyline; Top-k; Top-k dominating; Preference queries; Preference evaluation techniques 